\title{
Percepción del éxito en maratonistas en función de sus hábitos de entrenamiento Perception of success in marathoners depending on their training habits
}

\author{
*Antonio Zarauz-Sancho, **Francisco Ruiz-Juan, ***Gabriel Flores-Allende, **María Elena García Montes \\ *Azcona de Almería (España), **Universidad de Murcia (España), *** Universidad de Guadalajara (México)
}

Resumen. Se pretendía conocer qué relaciones predictivas presentaban diversas variables de entrenamiento sobre la percepción del éxito en una amplia muestra de 1795 corredores de fondo en ruta (1105 españoles, 690 mexicanos). De ellos, el 85.65\% fueron hombres con una edad de M=38.98 (DT=10.45), y el 14.35\% mujeres, con una edad M=37.88 (DT=9.80). La recogida de datos se llevó a cabo mediante un cuestionario que incluía la adaptación al castellano del Perception of Success Questionnaire (POSQ) y diversas variables de entrenamiento. Se obtuvieron valiosos datos de entrenamiento, que los valores en las orientaciones de meta de los corredores fueron medios y similares, así como dos sólidos modelos predictivos de las orientaciones de meta en función de sus hábitos de entrenamiento claramente diferenciados por sexos.

Palabras clave: Ego; tarea; deporte individual; POSQ.

Abstract. We pretended to find out which predictive relationships would be introduced by some training habits, concerning the winning perception of a broad sample of 1795 long-distance road runners (1105 Spanish, 690 Mexicans). Of them, 85.65\% were men, aged M=37.88 (SD=10.45) and 14.35\% were women, aged $\mathrm{M}=37.88$ ( $\mathrm{SD}=9.80$ ). The data was obtained by a questionnaire that containing adapting to Spanish the following: Perception of Success Questionnaire (POSQ) and some training habits. Valuable training data were obtained, that the values obtained on the goal orientations of the runners were moderate and similar, as well as two solid predictive models of the goal orientations according to their training habits clearly differentiated by sex.

Key words: Ego; task; individual sport; POSQ.

\section{Introduccion}

En la actualidad, existe una actividad física que no hace sino crecer significativamente en popularidad; la práctica de la carrera continua en general y el entrenamiento para la participación en carreras de larga distancia en ruta en particular. Las cifras así lo atestiguan; de hecho, en las últimas tres décadas, ya sea por el número de carreras de medio maratón o maratón que se organizan al año, o por el número de participantes en cada una de esas carreras, este incremento ha sido significativo, tanto en países anglosajones (Dawson \& Peco, 2004; Masters, Ogles \& Jolton, 1993; Ogles \& Masters, 2000), como hispanohablantes (Ruiz-Juan \& Zarauz, 2012; 2014; Ruiz-Juan, Zarauz \& FloresAllende, 2016a; Zarauz \& Ruiz-Juan, 2011).

Ya son numerosos los estudios que han analizado a esta creciente población de corredores de fondo en ruta, desde los que investigan sobre sus motivaciones (Masters, et al., 1993; Ogles \& Masters, 2000; Zarauz \& Ruiz-Juan, 2013a), a los que lo hacen sobre su adicción a correr (Chapman \& de Castro, 1990; Jaenes \& Caracuel, 2016; Ruiz-Juan \& Zarauz, 2012), el compromiso que sienten con su práctica deportiva (Dawson \& Peco, 2004; Horton \& Mack, 2000; Zarauz, Ruiz-Juan \& Flores-Allende, 2014), su ansiedad precompetitiva (Hammermeister \& Burton, 2001; Ruiz-Juan \& Zarauz, 2014; Ruiz-Juan, et al., 2016), e incluso, un compendio de factores que pueden influir en el rendimiento posterior en competición de los corredores (Buceta, López, PérezLlantada, Vallejo \& del Pino, 2003; Jaenes, Godoy \& Román, 2009; Larumbe, Pérez-Llantada \& López de la Llave, 2009).

Sin embargo, no abundan en la literatura investigaciones que analicen la percepción del éxito en el deporte en esta población. Desde la perspectiva de las metas de logro se ha construido uno de los modelos teóricos más utilizados para analizar y comprender diversas variables conductuales, cognitivas, e incluso, emocionales relacionadas con el logro en el deporte. La teoría de las Metas de Logro (Nichols, 1989) explica que la orientación al ego serelaciona con la creencia de que eléxito en el deporte se alcanza gracias a tener una mayor habilidad que los rivales y, a través incluso, del uso de técnicas engañosas. A su vez, el deportista con mayor orientación al ego, tiene como finalidad lograr un mayor estatus social respecto a los compañeros, obteniendo satisfacción con los resultados que proporcionan información sobre la posesión de mayor habilidad que los demás y mayor aprobación social, pero con menor diversión con su práctica deportiva.

Fecha recepción: 15-09-16. Fecha de aceptación: 21-01-17 Francisco Ruiz Juan

pacoruizjuan@gmail.com
Por el contrario, la orientación a la tarea se relaciona con los patrones motivacionales más adaptativos, consistentes en creer que el éxito en el deporte se consigue a través del esfuerzo personal, pensando que el deporte tiene como fin la formación y desarrollo personal. Así, el deportista con orientación a la tarea, siente la satisfacción con los resultados que informan sobre su progreso personal y, además, se relaciona con una mayor motivación autodeterminada y diversión con la práctica deportiva (Carlin, 2015).

En un análisis comparativo entre corredores de maratón con mayor y menor experiencia en esta carrera, Ogles y Masters (2000) concluyeron que los más jóvenes tenían una mayor orientación al ego y los más experimentados una mayor orientación a la tarea. Por su parte, Steimberg, Grieve y Glass (2001), obtuvieron que los mayores de 50 años tenían valores significativamente menos elevados que los jóvenes en orientación al ego. Continuando con la comparativa por edades en la población de maratonistas española, Ruiz-Juan y Zarauz (2011) obtuvieron que ambos sexos tenían una alta orientación a la tarea, que aumentaba con la edad.

Pero no sólo el sexo, la experiencia o la edad son variables que pueden mediar en la percepción del éxito de los maratonistas pues, recientemente, Ruiz-Juan et al. (2015), además de obtener valores medios en ambas orientaciones de meta sin diferencias significativas por sexo, obtuvieron que el hecho de tener entrenador incrementaba significativamente tanto la orientación al ego como la orientación a la tarea de todos los corredores varones, además de su menor edad.

Queda claro pues, por un lado, la escasez de investigaciones sobre las orientaciones de meta de los maratonistas y, por otro, la importancia y necesidad del estudio de este constructo psicológico tan analizado en otras poblaciones de deportistas. Como se ha demostrado en esta población y en otras similares, los hábitos de entrenamiento y la experiencia de los atletas pueden actuar como moderadores de su ansiedad (Ruiz-Juan, et al., 2016; Zarauz \& Ruiz-Juan, 2013b), de su motivación (Zarauz, Ruiz-Juan \& Flores-Allende, 2016), e incluso, de su dependencia a correr (Ruiz-Juan, Zarauz \& Flores-Allende, 2016b). Por ello, el objetivo de la presente investigación pasa por tratar de arrojar algo más de luz al respecto de qué variables de entrenamiento pueden ser predictoras de una u otra orientación disposicional de meta en una amplia muestra de corredores de fondo en ruta y sus diferencias por sexo, dado que aún no están suficientemente claras según la literatura consultada.

Se parte de la hipótesis de que, tanto hombres como mujeres, van a mostrar moderados niveles de percepción del éxito, tanto en la orientación al ego como en la orientación a la tarea, y que la mayoría de variables de entrenamiento que se van a analizar van a predecir una u otra orientación de meta. 


\section{Método}

\section{Participantes}

Se partió del total de corredores inscritos en los medios maratones de Almería y Elche (España) y Guadalajara (México) en 2010. Para asegurar que la muestra fuera representativa (error $\pm 3 \%$, intervalo de confianza 95.5\%), se empleó un diseño de muestreo estratificado por afijación proporcional teniendo en cuenta el sexo (86.65\% de hombres y 13.35\% de mujeres) y la edad. Se administró un cuestionario a 1060 corredores de fondo en ruta que participaron en los medios maratones deAlmería (30/01/2011), Elche(03/04/2011) y Guadalajara (20/02/11; 19/02/2012). Además, por medio de una página Web se obtuvieron las respuestas de 741 cuestionarios de corredores de maratón y medio maratón desde 01/03/11 a 20/03/13. Por tanto, la muestra aleatoria y voluntaria fue de 1795 (1105 españoles, 690 mexicanos) corredores de fondo en ruta que estaba compuesta por 1541 hombres (85.7\%) con rango de edad de 18 a 76 años $(M=38.98 ; D T=10.45)$ y 254 mujeres (14.35\%) con rango de edad de 18 a 69 años $(M=37.88 ; D T=9.80)$. Por tanto, se tiene una muestra representativa con un error del $\pm 2.30 \%$, a un intervalo de confianza del $95.5 \%$.

\section{Procedimiento}

En las carreras citadas anteriormente, se pidió permiso a la organización de la carrera mediante una carta en la que se explicaban los objetivos de la investigación, cómo se iba a realizar el estudio y se acompañaba un modelo del instrumento. El cuestionario fue administrado en un stand que se dispuso al efecto durante la recogida de dorsales de los atletas participantes el día previo a la carrera.

Para ampliar la muestra y obtener la más amplia y variada geográficamente en el territorio español, se solicitó colaboración al webmaster del principal foro de atletismo de España (http:// www.elatleta.com/foro/forum.php), al que se le pidió que colgara el cuestionario en la sección de corredores de ruta (http://www.retos.org/ encuesta/inicio.html).

En ambos casos, todos los sujetos fueron informados del objetivo del estudio, de la voluntariedad, absoluta confidencialidad de las respuestas y manejo de los datos, que no había respuestas correctas o incorrectas y se les solicitó que contestaran con la máxima sinceridad y honestidad. Además, se pidió que si habían contestado previamente este cuestionario no lo volvieran a hacer. Este trabajo posee el informe favorable de la Comisión de Bioética de la Universidad de Murcia.

Esta misma muestra y procedimiento ha sido empleada por los autores en otros estudios con diferentes objetivos, constructos psicológicos analizados, resultados y conclusiones (Ruiz-Juan \& Zarauz, 2014; Ruiz-Juan, Zarauz \& Flores-Allende, 2015; Zarauz \& Ruiz-Juan, 2013a; Zarauz, Ruiz-Juan \& Flores-Allende, 2014 y 2016).

\section{Instrumentos}

Se aplicó un cuestionario que estaba constituido por:

Cuestionario de Percepción de Éxito; versión española de Cervelló (1996) del Perception of Success Questionnaire (POSQ) de Roberts y Balagué(1991). Fue elaborado para medir la Orientación disposicional de las metas de logro en el contexto deportivo. Consta de 12 ítems, 6 de implicación disposicional en la tarea y otras 6 en el ego. La pregunta inicial que encabeza el cuestionario es «Siento éxito en el deporte cuando...». Las respuestas se recogen en escala tipo Likert que oscilan desde totalmente en desacuerdo (1) a totalmente de acuerdo (5).

Hábitos de entrenamiento: promedios de kilómetros entrenados a la semana, tiempo por entrenamiento diario, días entrenados a la semana, porcentaje de tiempo que entrena solo a la semana, años corriendo, tener entrenador, compañeros de entrenamiento e índice de masa corporal.

\section{Análisis de los datos}

Correlación entre las subescalas (coeficiente de Pearson), diferencias de medias por sexo ( $t$ Student) y regresión lineal multivariante, se realizaron con SPSS 20.0.

\section{Resultados}

\section{Estadística descriptiva}

Fueron medias las puntuaciones obtenidas en la percepción de éxito en el deporte por ego y tarea, en ambos sexos, siendo el valor de la orientación al ego ligeramente superior al de la tarea. No se han hallado diferencias estadísticamente significativas según el sexo (Tabla 1).

Las mujeres tienen entrenador en un porcentaje mayor (37.4\%) que los hombres (19.3\%), siendo estas diferencias significativas ( $\mathrm{p}<.001)$, al igual que dedican más minutos de entrenamiento en cada sesión, entrenan más días a la semana y lo hacen con mayor número de compañeros que los hombres. Por el contrario, siempre con diferencias estadísticamente significativas, los hombres corren más kilómetros semanales que las mujeres, entrenan mayor porcentaje de tiempo solos, llevan más años corriendo y tienen mayor IMC que las mujeres (Tabla 1).

\section{Tabla 1.}

Medias, $D T$, t, p-valor y $d$ de la percepción del éxito en el deporte (ego y tarea) y variables de hábitos de entrenamiento e historial atlético, según sexo (hombres $n=1541$; mujeres $n=254$ ). Ego Km/semana

\begin{tabular}{ccccccc}
$M_{\text {Hombres }}$ & $D T$ & $M_{\text {Mujeres }}$ & $D T$ & $t$ & $p$ & $d$ \\
\hline 3.18 & .71 & 3.27 & .70 & -1.93 & .054 & -.12
\end{tabular}

Minutos entrenamiento por sesión Días entrenamiento por semana $\%$ entrenamiento solo por semana Años corriendo

$\mathrm{N}^{\circ}$ compañeros entrenamiento

$\begin{array}{lllllll}2.98 & .78 & 2.99 & .72 & -.33 & .739 & -.01\end{array}$
$\begin{array}{lllllll}51.17 & 25.78 & 43.89 & 25.64 & 4.17 & 000 & -28\end{array}$

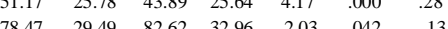
$\begin{array}{ccccccc}78.47 & 29.49 & 82.62 & 32.96 & -2.03 & .042 & -.13 \\ 4.60 & 1.25 & 4.90 & 1.32 & -3.60 & .000 & -.23\end{array}$ $\begin{array}{lllllll}67.76 & 34.01 & 56.88 & 37.73 & 4.64 & .000 & .30\end{array}$ $\begin{array}{lllllll}9.22 & 8.84 & 8.20 & 7.83 & 1.72 & .045 & .12\end{array}$ $\begin{array}{lllllll}2.92 & 5.71 & 5.04 & 9.57 & -4.89 & .000 & -.26\end{array}$ \begin{tabular}{llllllll} 
IMC & 23.74 & 3.63 & 21.54 & 3.76 & 12.23 & .000 & .59 \\
\hline
\end{tabular}

Relaciones de la percepción de éxito en el deporte con las variables de entrenamiento e historial atlético

La orientación al ego correlaciona positivamente, en hombres, con la orientación a la tarea, kilómetros que corren a la semana, minutos de entrenamiento por sesión, días de entrenamiento por semana y números de compañeros con los que entrena, y negativamente con años que lleva corriendo y tener entrenador. En las mujeres, correlaciona positivamente con orientación a la tarea, kilómetros que corren a la semana y días de entrenamiento por semana, y negativamente con tener entrenador (Tabla 2).

En hombres, la orientación a la tarea correlaciona positivamente con orientación al ego, kilómetros que corren a la semana, minutos de entrenamiento por sesión, días de entrenamiento por semana y números de compañeros con los que entrena y, negativamente, IMC y tener entrenador. En mujeres las correlaciones positivas y significativas fueron con la orientación al ego, kilómetros que corren a la semana y días de entrenamiento por semana y, negativamente, IMC y tener entrenador (Tabla 2).

Tabla 2.

Correlaciones entre de la percepción del éxito en el deporte (ego y tarea) con las variables de hábitos de entrenamiento e historial atlético según sexo (hombres $n=1541$; mujeres $n=254$ ).

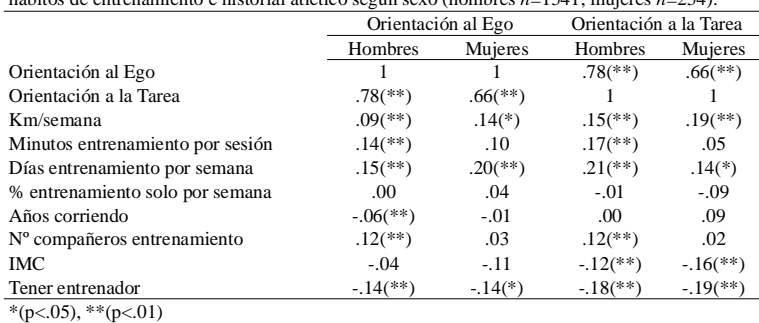

\section{Análisis regresivo multivariante}

Se realizó un análisis de regresión lineal multivariado, tratando de obtener unos modelos que explicasen la mayor parte posible de varianza. Se tomaron como variables dependientes las puntuaciones medias de la percepción de éxito en el deporte. Las variables predictoras fueron cada una de las variables de entrenamiento e historial atlético: promedios de kilómetros entrenados a la semana, tiempo por entrenamiento diario, 
días entrenados a la semana, porcentaje de tiempo que entrena solo a la semana, años corriendo, tener entrenador, compañeros de entrenamiento e IMC. Como variable de selección se consideró el sexo.

Se extrajo el valor $\mathrm{R}^{2}$ para explicar la varianza, Beta para explicar la predicción entre variables, F para ver si existe relación entre variables seleccionadas y su significatividad (Tabla 3). Se obtienen modelos consistentes, ya que explican entre casi la mitad y algo más de los dos tercios de la varianza en hombres y mujeres.

El modelo de la orientación al ego, en hombres, indicó que se podía predecir significativamente por puntuar alto en orientación a la tarea, porcentaje de tiempo que entrena solo a la semana, números de compañeros con los que entrena e IMC, y por puntuar bajo en años que lleva corriendo (varianza: 62.4\%). En las mujeres, el modelo fue bastante diferente a los hombres, ya que la predicción fue por puntuar alto en orientación a la tarea, días de entrenamiento a la semana, porcentaje de tiempo que entrena solo a la semana, y por puntuar bajo en años que lleva corriendo (varianza: 69.1\%).

Elmodelo de orientación a la tarea, en hombres, indicó que se podía predecir significativamente por puntuar alto en orientación al ego, días de entrenamiento a la semana y años que lleva corriendo, así como por puntuar bajo en IMC y tener entrenador (varianza: 63.2\%). En las mujeres, el modelo es algo diferente a los hombres ya que la predicción fue por puntuar alto en orientación al ego, porcentaje de tiempo que entrena solo a la semana y años que lleva corriendo, así como por puntuar bajo en IMC y tener entrenador.

\section{Tabla 3.}

Análisis Regresivo Lineal Multivariado; modelos que predicen significativamente de la

percepción del éxito en el deporte (ego y tarea) por sexos en función de variables de hábitos de entrenamiento (hombres $n=1541$; mujeres $n=254$ ).

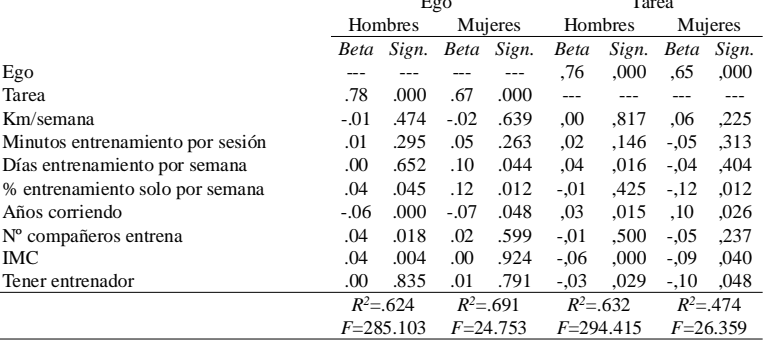

\section{Discusión y conclusiones}

Los valores obtenidos en la presente investigación, tanto en orientación al ego como a la tarea, han sido similares y moderados, como sucedió en la investigación con maratonistas de Ruiz-Juan et al. (2015). Por el contrario, en otras actividades deportivas, los valores obtenidos en la orientación al ego fueron medios y en la orientación a la tarea altos o muy altos (Cecchini, González, Carmona \& Contreras, 2004; Cecchini, González \& Montero, 2008; González-Cutre, Sicilia \& Moreno, 2006; Ruiz-Juan, Gómez-López, Pappous, Alacid \& Flores-Allende, 2010; Standage \& Treasure, 2002; Zizzi, Keeler \& Watson II, 2006).

También sucede esto en otra población del mismo deporte similar a la de los corredores de ruta, la de los atletas veteranos especialistas en pruebas de pista, quienes muestran un equilibrio en los moderados valores obtenidos en sus orientaciones de meta (Zarauz \& Ruiz-Juan, 2015 y 2016). Sin embargo, es importante destacar que, como en la mayoría de poblaciones ya mencionadas de otros deportes, sus motivaciones son principalmente autodeterminadas.

La explicación a esta menor orientación a la tarea respecto a otros deportes, incluso a otras especialidades dentro del mismo deporte, podría venir dada por la altísima puntuación que normalmente obtienen en la motivación de salud los corredores de ruta (Zarauz \& Ruiz-Juan, 2013a; Zarauz, Ruiz-Juan, Flores-Allende \& Arufe, 2016), más que por lo divertido en sí de esta práctica deportiva respecto a otras. De hecho, los atletas veteranos especialistas en pruebas de pista, obtienen las mayores puntuaciones en diversión y motivación intrínseca a las experiencias estimulantes (Zarauz \& Ruiz-Juan, 2016) por lo gratificante que les resulta ejecutar con maestría una y otra vez los complejos movimientos de su especialidad atlética, fundamentalmente en competición, algo que no sucede en corredores de resistencia en ruta, dado lo monótono y simple de su ejecución práctica.

Tanto el análisis correlacional como el regresivo, aportan solidez a este equilibrio en los moderados valores obtenidos en las orientaciones de meta de los corredores, ya que la orientación al ego correlaciona y se predice por la orientación a la tarea y viceversa en ambos sexos.

En cuanto a los modelos predictivos obtenidos en el análisis regresivo, comenzar destacando que ni los kilómetros entrenados por semana ni los minutos entrenados por sesión fueron predictores de ninguna de las dos orientaciones de meta en ambos sexos. Esto puede quedar explicado si aludimos nuevamente a lo monótono o simple que resulta la carrera continua, frente a lo divertido o complejo que puedan resultar otras especialidades deportivas.

Sin embargo, otra variable cuantitativa de entrenamiento, el mayor número de días de entrenamiento por semana, en las mujeres fue predictor de una mayor orientación al ego, mientras que en los hombres lo fue de la orientación a la tarea, evidenciando una vez más con claridad las diferencias por sexo y la necesidad de analizar ambos géneros por separado. En una investigación similar, Ruiz-Juan et al. (2016a) concluyeron que entrenar más días a la semana, tanto en hombres como en mujeres, aumentaba la autoconfianza de los corredores. En las mujeres, que entrenan casi cinco días a la semana de media, parece ser que esto las hace tener más confianza en superar a sus rivales en competición. Los hombres, que entrenan un número de días significativamente menor que las mujeres, sin embargo, lo hacen más con una orientación de meta a la tarea.

El porcentaje de tiempo entrenando en solitario y el mayor IMC, por el contrario, sí que ha resultado ser en ambos sexos predictor de una mayor orientación al ego, e incluso, en las mujeres, de una menor orientación a la tarea. Este dato podría quedar explicado porque los corredores que buscan la compañía de otros, tienen motivaciones más altas de afiliación (Zarauz, et al., 2016), mientras que los que lo hacen más en solitario, buscan más un mayor rendimiento que socializar con otros corredores.

Del análisis regresivo, por último, podemos extraer que el tener entrenador es predictor de una menor orientación a la tarea, tanto en hombres como en mujeres. Apriori, parece evidenciarse que, en ambos sexos, cuando se busca el asesoramiento de un entrenador es más para lograr éxitos deportivos y victorias sobre los rivales, que por el mero disfrute de esta práctica deportiva en sí. Sin embargo, según Ruiz-Juan et al. (2016a), sólo los corredores varones con entrenador muestran menos autoconfianza en sus posibilidades, e incluso, menor motivación de superación de metas personales-competición y de reconocimiento (Zarauz, et al., 2016), lo cual podría quedar explicado por el menor número de días que entrenan a la semana respecto a las mujeres.

\section{Referencias}

Buceta, J.M., López, A., Pérez-Llantada, M.C., Vallejo, M., \& Del Pino, M.D. (2003). Estado psicológico de los corredores populares de maratón en los días anteriores a la prueba. Psicothema, 15, 273-277.

Carlin, M. (2015). El abandono de la práctica deportiva, motivacióny estados de ánimo en deportistas. Sevilla: Wanceulen.

Cecchini, J.A., González, C., Carmona, A.M., \& Contreras, O. (2004) Relaciones entre el clima motivacional, la orientación de meta, la motivación intrínseca, la auto-confianza, la ansiedad y el estado de ánimo en jóvenes deportistas. Psicothema, 16(1), 104-109.

Cecchini, J.A., González, C. \& Montero, J. (2008). Participación en el deporte, orientación de metas y funcionamiento moral. Revista Latinoamericana de Psicología, 40(3), 497-509.

Cervelló, E. (1996). La motivación y el abandono deportivo desde la perspectiva de las metas de logro. Tesis Doctoral. Universitat de València, Valencia.

Chapman, C.L., \& De Castro, J.M. (1990). Running addiction: 
measurement and associated psychological characteristics. The Journal of Sports Medicine and Physical Fitness, 30, 283-290.

Dawson, K.A. \& Peco, J. (2004). Exercise motivation, commitment, addiction and gender. Medicine \& Science in Sports \& Exercise, 36, 64-65.

González-Cutre, D., Sicilia, A., \& Moreno, J.A. (2006). Predicción de las razones de responsabilidad para ser disciplinado en el deporte a través de las orientaciones de meta y los climas motivacionales. En M.A. González, J.A. Sánchez y A. Areces (Eds.), IV Congreso de la Asociación Española de Ciencias del Deporte (pp. 736-739). A Coruña: Xunta de Galicia.

Hammermeister, J., \& Burton, D. (2001). Stress, appraisal, and coping revisited: examining the antecedents of competitive state anxiety with endurance athletes. Sport Psychologist, 15(1), 66-90.

Horton, R.S., \& Mack, D.E. (2000). Athletic identity in marathon runners: functional focus or dysfunctional commitment? Journal of Sport Behavior, 23, 101-119.

Jaenes. J.C., \& Caracuel. J.C. (2016). Maratón. Preparación psicológica para el entrenamientoy la competición. Córdoba:Almuzara.

Jaenes, J.C., Godoy, D., \& Román, F.M. (2009). Personalidad resistente en maratonianos: un estudio sobre el control, compromiso y desafío de corredoras y corredores de maratón. Revista de Psicología del Deporte, 18(2), 217-234.

Masters, K.S., Ogles B.M., \& Jolton, J.A. (1993). The development of an instrument to measure Motivation for Marathon running: the Motivations of Marathoners Scales (MOMS). Research Quarterly for Exercise and Sport (RQES), 64, 134-143.

Larumbe, E., Pérez-Llantada, C., \& López de la Llave,A. (2009). Características del estado psicológico de los corredores populares de maratón. Revista de Psicología del deporte, 18(2), 151-163.

Ogles, B.M., \& Masters, K.S. (2000). Older vs. younger adult male marathon runners: participative motives and training habits. Journal of Sport Behavior, 23(2), 130-143.

Roberts, G., \& Balagué, G (1991, septiembre). The development and validation of the Perception of Success Questionnaire. Paper presented at the FEPSAC Congress, Cologne, Germany.

Ruiz-Juan, F., Gómez-López, M., Pappous, A., Alacid, F., \& FloresAllende, G. (2010). Dispositional goal orientation, beliefs about the causes of success and intrinsic satisfaction in Young elite paddelers. Journal of Human Kinetics, 26, 123-136. DOI: http://dx.doi.org/ 10.2478/v10078-010-0056-8

Ruiz-Juan F., \& Zarauz,A. (2011). Validación de la versión española de las Motivations of Marathoners Scales (MOMS). Revista Latinoamericana de Psicología, 43(1), 139-156.

Ruiz-Juan F., \& Zarauz, A. (2012). Variables que hacen adicto negativamente a correr al maratoniano español. Retos, 21, 38-42.

Ruiz-Juan, F., \& Zarauz, A. (2014). Ansiedad en maratonianos en función de variables socio-demográficas. Retos, 25, 5-8.

Ruiz-Juan, F., Zarauz, A., \& Flores-Allende (2015).Percepción del éxito en corredores de fondo en ruta en función de variables sociodemográficas. Retos, 27, 136-139.

Ruiz-Juan, F., Zarauz, A., \& Flores-Allende, G. (2016a). Ansiedad precompetitiva en corredores de fondo en ruta en función de sus variables de entrenamiento. Retos, 30, 110-113.

Ruiz-Juan, F., Zarauz, A., \& Flores-Allende, G. (2016b). Dependencia al entrenamiento y competición en corredores de fondo en ruta. Cultura, Ciencia y Deporte, 32(11), 127-133.

Standage, M., \& Treasure, D.C. (2002). Relationship among achievement goal orientations and multidimensional situational motivation in physical education. British Journal of Education Psychology, 72, 87-103. DOI: http://dx.doi.org/10.1348/000709902158784

Steimberg, G., Grieve, F.G., \& Glass, B. (2001). Achievement Goals across the Lifespam. Journal of Sport Behavior, 24, 298-306.

Zarauz,A., \& Ruiz-Juan, F. (2011). Compromiso y adicción negativa al entrenamiento y competición de los maratonianos. Revista Internacional de Medicinay Ciencias de la Actividad Física yel Deporte, 11(44), 817-834.

Zarauz,A., \& Ruiz-Juan, F. (2013a). Motivaciones de los maratonianos según variables socio-demográficas y de entrenamiento. Retos, 24, 50-56.

Zarauz, A., \& Ruiz-Juan, F. (2013b). Variables predictoras de la ansiedad en atletas veteranos españoles. Retos, 23, 29-32.

Zarauz, A., \& Ruiz-Juan, F. (2015). Factores determinantes de la motivación en atletas veteranos. Revista Latinoamericana de Psicología, 47(1), 34-42. DOI: http://dx.doi.org/10.1016/S01200534(15)30004-2

Zarauz, A., \& Ruiz-Juan, F. (2016). Motivación, satisfacción, percepción y creencias de las causas del éxito en atletas veteranos españoles. Revista Iberoamericana de Psicología del Ejercicio y el Deporte, 11(1), 37-46.

Zarauz, A., Ruiz-Juan, F., \& Flores-Allende (2014). Compromiso con el entrenamiento y competición de los maratonianos según variables socio-demográficas. Retos, 26, 118-121.

Zarauz,A., Ruiz-Juan, F., \& Flores-Allende(2016). Modelos predictores de la motivación en corredores de fondo en ruta en función de sus hábitos de entrenamiento. Revista Iberoamericana de Psicología del Ejercicio y el Deporte, 11(2), 185-192.

Zarauz,A., Ruiz-Juan, F., Flores-Allende, G., \& Arufe, V. (2016). Variables predictoras de la percepción del éxito: aspectos diferenciales en corredores de ruta. Revista Internacional de Medicina y Ciencias de la Actividad Física y el Deporte, 16(63), 601-615. DOI: http:// dx.doi.org/10.15366/rimcafd2016.63.012

Zizzi, S., Keeler, L.A., \& Watson II, J.C. (2006). The Interaction of Goal Orientation and Stage of Change on Exercise Behavior in College Students. Journal of Sport Behavior, 29(1), 96-110.
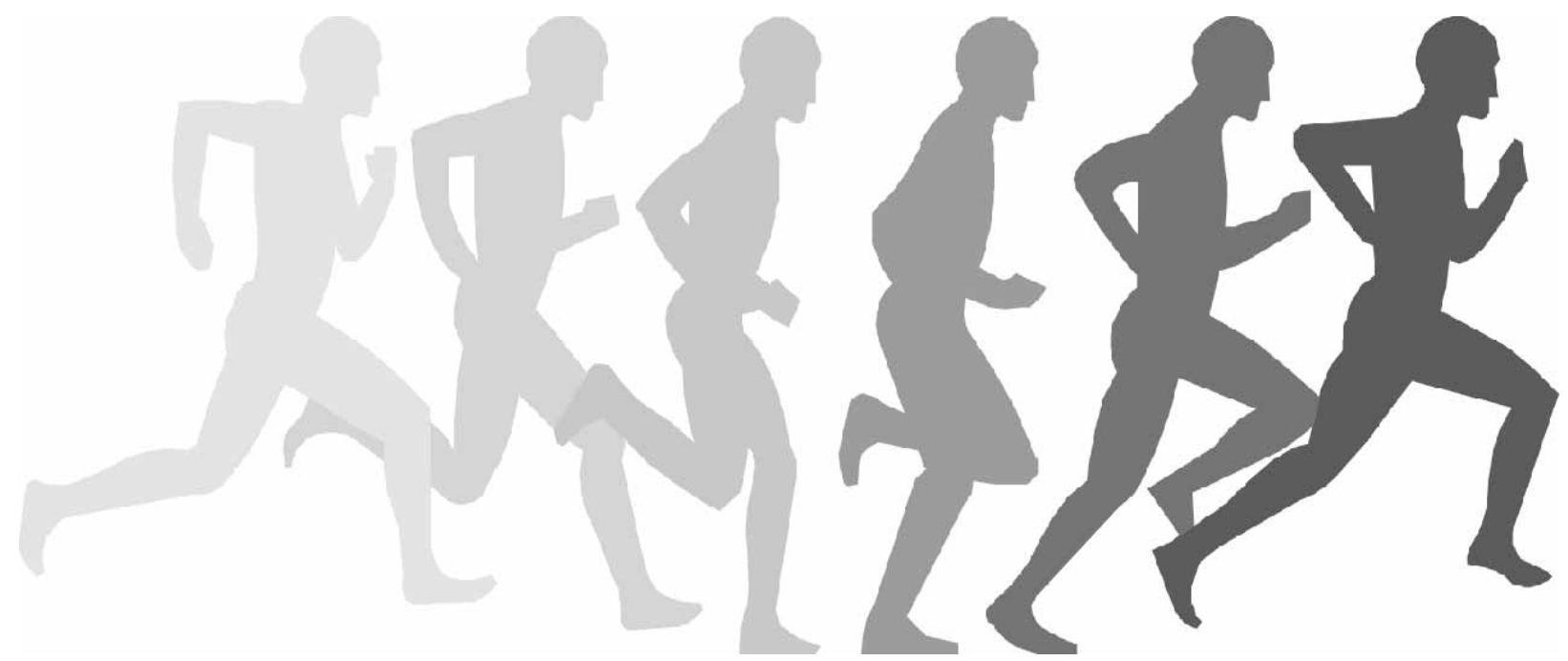